\title{
Serous adenocarcinoma of the sigmoid mesentery arising in cystic endosalpingiosis
}

\section{A McCoubrey, O Houghton, K McCallion, W G McCluggage}

J Clin Pathol 2005;58:1221-1223. doi: 10.1136/icp.2005.027946

\begin{abstract}
This case report describes a Mullerian serous adenocarcinoma arising within a multoloculated cyst lined by ciliated serous-type epithelium located in the sigmoid mesentery. Twenty years previously the patient underwent a hysterectomy, bilateral salpingo-oophorectomy, and omentectomy. The ovaries contained bilateral serous cystadenofibromas, and multiple cysts lined by ciliated serous-type epithelium were present in the omentum. The resection specimen 20 years later contained a $14 \mathrm{~cm}$ multiloculated cyst located in the sigmoid mesentery. This was lined largely by benign ciliated serous-type epithelium but a focus of well differentiated serous adenocarcinoma projected into the lumen. Two further peritoneal cysts were present, both of which were lined by ciliated serous-type epithelium. There was a coincidental renal cell carcinoma. This is a unique case of multiple omental, peritoneal, and retroperitoneal cysts (classified as cystic endosalpingiosis), one of which developed a focus of serous adenocarcinoma. Although rarely serous adenocarcinomas, similar to those occurring within the ovary, arise in the retroperitoneum, this is the first reported occurrence in association with a pre-existing benign lesion.
\end{abstract}

S erous adenocarcinoma is the most common epithelial ovarian malignancy. Identical tumours arising as primary peritoneal lesions are well described, and are known as primary peritoneal serous carcinomas. ${ }^{12}$ However, primary serous adenocarcinoma of the retroperitoneum is very rare with, as far as we are aware, only four previously reported cases in the English language literature. ${ }^{3-6}$ We describe a case of a retroperitoneal serous adenocarcinoma arising in the sigmoid mesentery in a women who had a hysterectomy, bilateral salpingo-oophorectomy, and omentectomy 20 years earlier. Bilateral ovarian serous cystadenofibromas were present and multiple cysts lined by ciliated serous-type epithelium, in keeping with cystic endosalpingiosis, ${ }^{7-9}$ were present in the omentum in the original specimen and in the peritoneum and sigmoid mesentery 20 years later. The large cyst in the sigmoid mesentery contained a focus of well differentiated serous adenocarcinoma projecting into the lumen. As far as we are aware, this is the first reported case of malignant transformation of cystic endosalpingiosis and the first documented example of a retroperitoneal serous adenocarcinoma arising within a preexisting benign lesion.

"This is the first reported case of malignant transformation of cystic endosalpingiosis"

\section{CASE REPORT}

A 72 year old woman was being followed up for colitis and colonic polyps. At routine review, she was found to be anaemic and described weight loss of $12 \mathrm{~kg}$. Abdominal examination was unremarkable but a digital rectal examination revealed a palpable mass anteriorly, which appeared to be extrinsic to the rectum.

Her past history included a total abdominal hysterectomy, bilateral salpingo-oophorectomy, and omentectomy 20 years previously for a fibroid uterus and bilateral ovarian masses. Multiple small cysts were noted within the omentum at that time.

At this review, an ultrasound scan of the abdomen showed a $6 \mathrm{~cm}$ solid lesion in the lower pole of the right kidney. A large mass was present in the pelvis, which was indenting the bladder wall. A computed tomography scan of the abdomen and pelvis confirmed the presence of a large mainly cystic mass arising from the pelvis, with a solid component inferiorly. The presence of a mass within the right kidney was confirmed. The serum CAl25 concentration was 72 units/ml.

A right nephrectomy and pelvic cystectomy were performed. At surgery, a $14 \mathrm{~cm}$ cystic mass was adherent to and indistinguishable from the sigmoid mesentery. The cyst was removed en bloc with rectum and sigmoid colon with primary anastomosis and defunctioning loop ileostomy. There were two further small $1-2 \mathrm{~cm}$ peritoneal cysts, one in the left lower quadrant and one in the right upper quadrant, which were removed.

\section{PATHOLOGICAL FINDINGS}

The slides of the original specimen were reviewed. This confirmed bilateral ovarian serous cystadenofibromas, measuring $15 \mathrm{~cm}$ and $8 \mathrm{~cm}$ in maximum dimension. These contained multiple cysts lined by benign ciliated serous-type epithelium set in a fibrous stroma (fig 1). In addition, multiple cysts-the largest measuring $1.5 \mathrm{~cm}$ in maximum dimension-were present in the omentum lined by ciliated serous-type epithelium. The uterus contained benign leiomyomas.

The second specimen consisted of a right nephrectomy together with a $30 \mathrm{~cm}$ length of sigmoid colon and rectum. A $6 \mathrm{~cm}$ tumour was located in the lower pole of the right kidney. A $14 \mathrm{~cm}$ multiloculated thick walled cystic mass was located in the mesentery of the sigmoid colon. A $4 \mathrm{~cm}$ solid white coloured nodule was present at one aspect projecting into the lumen of the cyst. Two thin walled cysts, measuring 2 and $1 \mathrm{~cm}$ in maximum dimension, were submitted separately.

Histology of the renal tumour showed a clear cell carcinoma, which invaded the renal vein (stage 3B). The cyst within the sigmoid mesentery was largely lined by a

Abbreviations: $\mathrm{CK}$, cytokeratin 


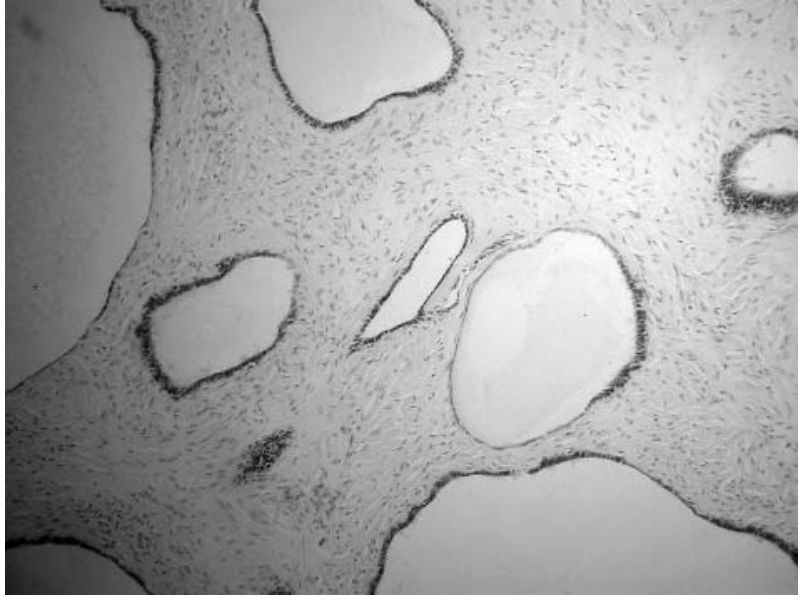

Figure 1 Ovarian serous cystadenofibroma containing cysts lined by ciliated epithelium within a fibrous stroma.

single layer of benign ciliated serous-type epithelial cells with surrounding fibrous tissue (fig 2). In areas, the epithelial lining was eroded and replaced by haemosiderin laden macrophages. Endometrial-type stroma was not present. The solid nodule was composed of a complex papillary and glandular arrangement of cuboidal cells with mild to moderate nuclear atypia, in keeping with a well differentiated serous adenocarcinoma (fig 3). This was confined within the lumen of the cyst with no invasion of the wall. Areas of transition could be seen between the benign ciliated lining and the well differentiated serous adenocarcinoma.

Immunohistochemically, the tumour cells were positive for cytokeratin 7 (CK7), Wilms tumour gene (WT1), the oestrogen receptor, and the progesterone receptor, but were negative for CK20. The two smaller cysts were lined by ciliated serous-type epithelial cells.

\section{DISCUSSION}

Primary retroperitoneal epithelial neoplasms are rare, occurring almost exclusively in women. They may be of serous or mucinous type and, similar to their ovarian counterparts, may be benign, borderline, or malignant. Serous adenocarcinomas in this area are exceptionally rare, with only four previously reported cases, ${ }^{3-6}$ one of which occurred in an 11 year old girl. ${ }^{6}$ Morphologically, these tumours are identical to ovarian serous adenocarcinomas, but are not attached to the ovary. This is the first report of a serous adenocarcinoma arising in this location within a pre-existing benign cystic lesion. There are several theories regarding the histogenesis of these neoplasms. They could arise from heterotopic ovarian tissue but ovarian remnants have not been found within the tumours. A more plausible explanation is an origin from the secondary Mullerian system, as described by Lauchlan. ${ }^{10}$

In our case, the adenocarcinoma arose within a benign cyst lined by ciliated serous-type epithelium. We prefer to classify this as cystic endosalpingiosis rather than a pre-existing benign extraovarian serous cystadenoma because multiple lesions were present in both specimens. Endosalpingiosis is defined as the presence of benign ciliated serous-type epithelium outside the ovary and fallopian tube, the most common locations being the peritoneum and omentum. ${ }^{11}{ }^{12}$ Usually, endosalpingiosis is an incidental microscopic finding but rarely this may be cystic, forming a unilocular or multiloculated cystic mass, referred to as florid cystic endosalpingiosis. ${ }^{7-9}$ Endosalpingiosis is especially common in association with serous tumours of the ovary, probably

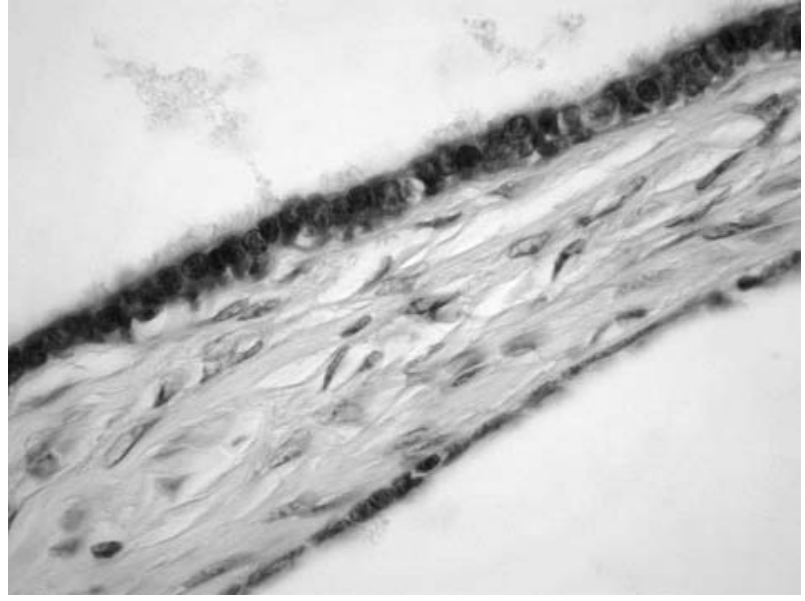

Figure 2 Cystic lesion lined by a single layer of ciliated serous-type epithelial cells.

representing a "field change" effect of the secondary Mullerian system. ${ }^{11}$ In our patient, bilateral ovarian serous cystadenofibromas were removed 20 years previously. At this time, several omental cysts lined by ciliated epithelium were present, in keeping with cystic endosalpingiosis. In addition, there were two further foci of peritoneal cystic endosalpingiosis in the second resection specimen separate from the large mass within the sigmoid mesentery.

"Optimal treatment for a serous adenocarcinoma arising in the retroperitoneum has not been established because of the rarity of these neoplasms"

We prefer to categorise the nodule projecting into the cyst lumen in the sigmoid mesentery as a well differentiated serous adenocarcinoma confined within the cyst, rather than a serous borderline tumour or an intraepithelial serous carcinoma. The overall architecture was not that of a serous borderline tumour but rather of a well differentiated serous adenocarcinoma. The term intraepithelial serous carcinoma, although used by some authors, is not in widespread use. The immunohistochemical features of the adenocarcinoma are entirely in keeping with a Mullerian neoplasm with positivity for CK7, the oestrogen receptor, and the progesterone receptor and negative CK20 staining. In addition, there was nuclear Wilms tumour gene (WT1) positivity, as is seen in

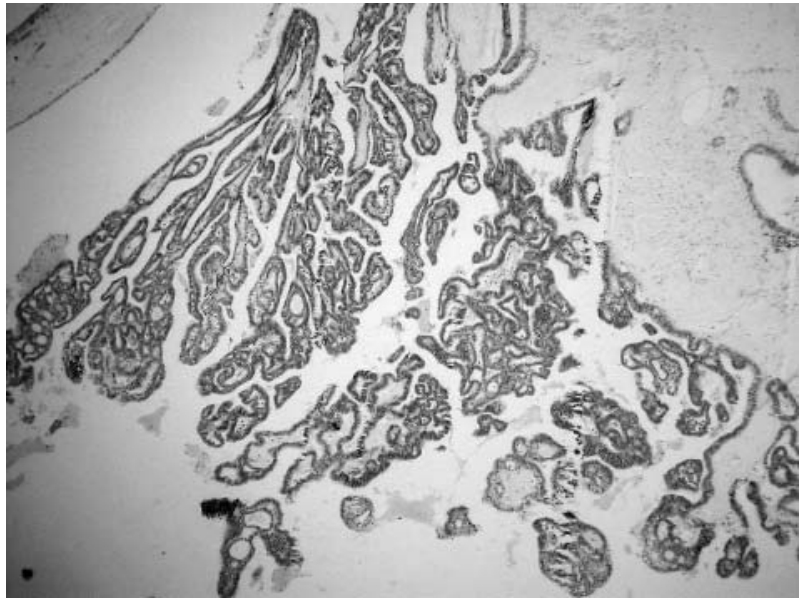

Figure 3 Well differentiated serous adenocarcinoma projecting into the lumen of the cystic lesion. 


\section{Take home messages}

- We report a unique case of a serous adenocarcinoma arising in the sigmoid mesentery within pre-existing cystic endosalpingiosis

- Although benign papillary proliferations have rarely been described in endosalpingiosis, this is the first report of malignant transformation of this benign condition

- Malignant transformation occurred over a long time period-multiple foci of endosalpingiosis were present in a resection 20 years previously

almost all primary ovarian and peritoneal serous carcinomas. ${ }^{13-15}$ This provides further evidence for a Mullerian, specifically a serous, phenotype.

Optimal treatment for a serous adenocarcinoma arising in the retroperitoneum has not been established because of the rarity of these neoplasms. Complete tumour resection is the mainstay of treatment. Adjuvant chemotherapy may be considered in patients with invasion of adjacent organs or in the presence of regional lymph node metastases. Combination chemotherapy should be based on regimens used for ovarian epithelial malignancies. In this case, ovarian-type chemotherapy was not administered because the carcinoma was small and well differentiated, and was confined within the lumen of a pre-existing cystic lesion. This is analogous to the situation with a small well differentiated serous adenocarcinoma within and confined to the ovary (stage 1A), where adjuvant chemotherapy would not be given. Moreover, in our present case a coincidental renal cell carcinoma was present, and the ultimate prognosis will probably depend on the renal tumour.

In summary, we report a unique case of a serous adenocarcinoma arising in the sigmoid mesentery within pre-existing cystic endosalpingiosis. Although benign papillary proliferations have rarely been described in endosalpingiosis, ${ }^{16}$ this is the first report of malignant transformation of this benign condition. Malignant transformation occurred over a long time period in that multiple foci of endosalpingiosis were present in the resection 20 years previously.

\section{Authors' affiliations}

A McCoubrey, K McCallion, Department of Surgery, Ulster Hospital, Dundonald BT16 ORH, Northern Ireland

O Houghton, W G McCluggage, Department of Pathology, Royal Group of Hospitals Trust, Grosvenor Road, Belfast BT12 6BL, Northern Ireland

Correspondence to: Dr W G McCluggage, Department of Pathology, Royal Group of Hospitals Trust, Grosvenor Road, Belfast BT12 6BL, Northern Ireland; glenn.mcccluggage@bll.n-i.nhs.uk

Accepted for publication 24 March 2005

\section{REFERENCES}

1 Ben-Baruch G, Sivan E, Moran O, et al. Primary peritoneal serous papillary carcinoma: a study of 25 cases and comparison with stage III-IV ovarian papillary serous carcinoma. Gynecol Oncol 1996;60:393-6.

2 Wick MR, Mills SE, Dehner LP, et al. Serous papillary carcinomas arising from the peritoneum and ovaries: a clinicopathologic and immunohistochemical comparison. Int J Gynecol 1989;8:179-88.

3 Caruncho M, Pombo F, Arnal-Monreal F. Primary retroperitoneal serous cystadenocarcinoma of "ovarian-type": US and CT findings. Eur J Radiol 1993:17:115-16.

4 Kaku M, Ohara N, Seima Y, et al. A primary retroperitoneal serous cystadenocarcinoma with clinically aggressive behavior. Arch Gynecol Obstet 2004;270:302-6.

5 Kurosaki Y, Kuramoto K. Case report: serous cystadenocarcinoma of the retroperitoneum: CT and sonographic appearance. Clin Radiol 1998;53:916-18.

6 Ulbright TM, Morley DJ, Roth LM, et al. Papillary serous carcinoma of the retroperitoneum. Am J Clin Pathol 1983;79:633-7.

7 Clement PB, Young RH. Florid cystic endosalpingiosis with tumor-like manifestations: a report of four cases including the first reported cases of transmural endosalpingiosis of the uterus. Am J Surg Pathol 1999;23:166-75.

8 McCluggage WG, Weir PE. Paraovarian cystic endosalpingiosis in association with tamoxifen therapy. J Clin Pathol 2000;53:161-2.

9 Heatley MK, Russell P. Florid cystic endosalpingiosis of the uterus. J Clin Pathol 2001;54:399-400.

10 Lauchlan SC. The secondary Mullerian system. Obstet Gynecol Surv 1972;27:133-46.

11 Zinsser KR, Wheeler JE. Endosalpingiosis in the omentum: a study of autopsy and surgical material. Am J Surg Pathol 1982;6:109-17.

12 McCluggage WG, Clements WD. Endosalpingiosis of the colon and appendix. Histopathology 2001;39:645-6.

13 McCluggage WG. WT1 is of value in ascertaining the site of origin of serous carcinomas within the female genital tract. Int J Gynecol Pathol 2004; 23:97-9.

14 Al-Hussaini M, Stockman A, Foster $\mathrm{H}$, et al. WT-1 assists in distinguishing ovarian from uterine serous carcinoma and in distinguishing between serous and endometrioid ovarian carcinoma. Histopathology 2004;44:109-15.

15 Shimizu M, Toki T, Takagi Y, et al. Immunohistochemical detection of the Wilms' tumor gene (WT1) in epithelial ovarian tumors. Int J Gynecol Pathol 2000;19:158-63.

16 McCluggage WG, O'Rourke D, McElhenney C, et al. Mullerian papilloma-like proliferation arising in cystic pelvic endosalpingiosis. Hum Pathol 2002;33:944-6. 